\title{
Airborne Position and Orientation System for Aerial Remote Sensing
}

\author{
Jianli Li, ${ }^{1,2}$ Jiancheng Fang, ${ }^{1}$ Zhaoxing Lu, ${ }^{1,3}$ and Lijian Bai ${ }^{1}$ \\ ${ }^{1}$ School of Instrument Science and Opto-Electronic Engineering, Beijing University of Aeronautics and Astronautics, \\ Beijing 100191, China \\ ${ }^{2}$ The National Key Lab of Satellite Navigation System and Equipment Technology, Shijiazhuang 050081, China \\ ${ }^{3}$ Xian Institute of Hi-Tech, Xian 710025, China
}

Correspondence should be addressed to Jianli Li; lijianli@buaa.edu.cn

Received 5 May 2016; Accepted 22 December 2016; Published 9 February 2017

Academic Editor: Mahmut Reyhanoglu

Copyright (C) 2017 Jianli Li et al. This is an open access article distributed under the Creative Commons Attribution License, which permits unrestricted use, distribution, and reproduction in any medium, provided the original work is properly cited.

\begin{abstract}
The airborne Position Orientation System (POS) can accurately measure space-time reference information and plays a vital role in aerial remote sensing system. It may be applied in a direct georeference system for optical camera and a motion imaging system for Synthetic Aperture Radar (SAR), which further advances efficiency and quality of imaging sensors. In this paper, the operation principle and components of airborne POS are introduced. Some key technologies of airborne POS are summarized. They include the error calibration and compensation, initial alignment, lever arm error modeling, time synchronization, and integrated estimation method. A high precision airborne POS has been developed and applied to a variety of aerial remote sensing systems.
\end{abstract}

\section{Introduction}

The airborne Position Orientation System (POS) is an inertial navigation system (INS) and Global Navigation Satellite System (GNSS) integrated measurement device dedicated to aerial remote sensing system. It has become universal device used for various imaging sensors $[1,2]$ comprising Synthetic Aperture Radar (SAR), scanning laser system [3], multispectral or hyperspectral scanners, digital or film cameras, and so on [4]. It can accurately provide space-time reference including velocity, position, and attitude solutions at the exact moment of data capture for optical camera. Thus, it is used in a direct georeference system and directly relates the data of imaging sensors to mapping frame independent of the Ground Control Points (GCP). Therefore, it can significantly improve the mapping efficiency and respond capacities, especially in some region where the GCP may be unavailable. On the other hand, the airborne POS can be used in motion compensation system for airborne SAR and achieves the ability to eliminate phase distortion of the radar return signals induced by antenna motion. Absence of POS will severely degrade the SAR image $[5,6]$. The compensation of antenna phase center is imperative [7]. The airborne POS has become a key technology to further advance efficiency and quality of aerial remote sensing system $[8,9]$.

The airborne POS differ from the existing INS/GNSS integrated system in part. Besides the requirements of small, lightweight IMU, it need accurately measure motion of the imaging sensor with high frequency and smooth velocity, position, and attitude solution in real-time and postprocess. Thus, some special key technologies comprising error calibration and compensation, initial alignment, time synchronization, lever arm compensation, postprocessing arithmetic, and so on determine POS's performance and have been introduced in this paper. An airborne POS is developed and tested in a variety of aerial remote sensing systems.

\section{Principle and Components of Airborne POS}

The airborne POS mainly includes four components: Inertial Measurement Unit (IMU), differential GNSS, POS Computer System (PCS) and postprocessing software shown in Figure 1 [10].

The inertial navigation system has a superior short term performance, but there is a long time drift error. The GNSS has good long-term accuracy but suffers from short term 


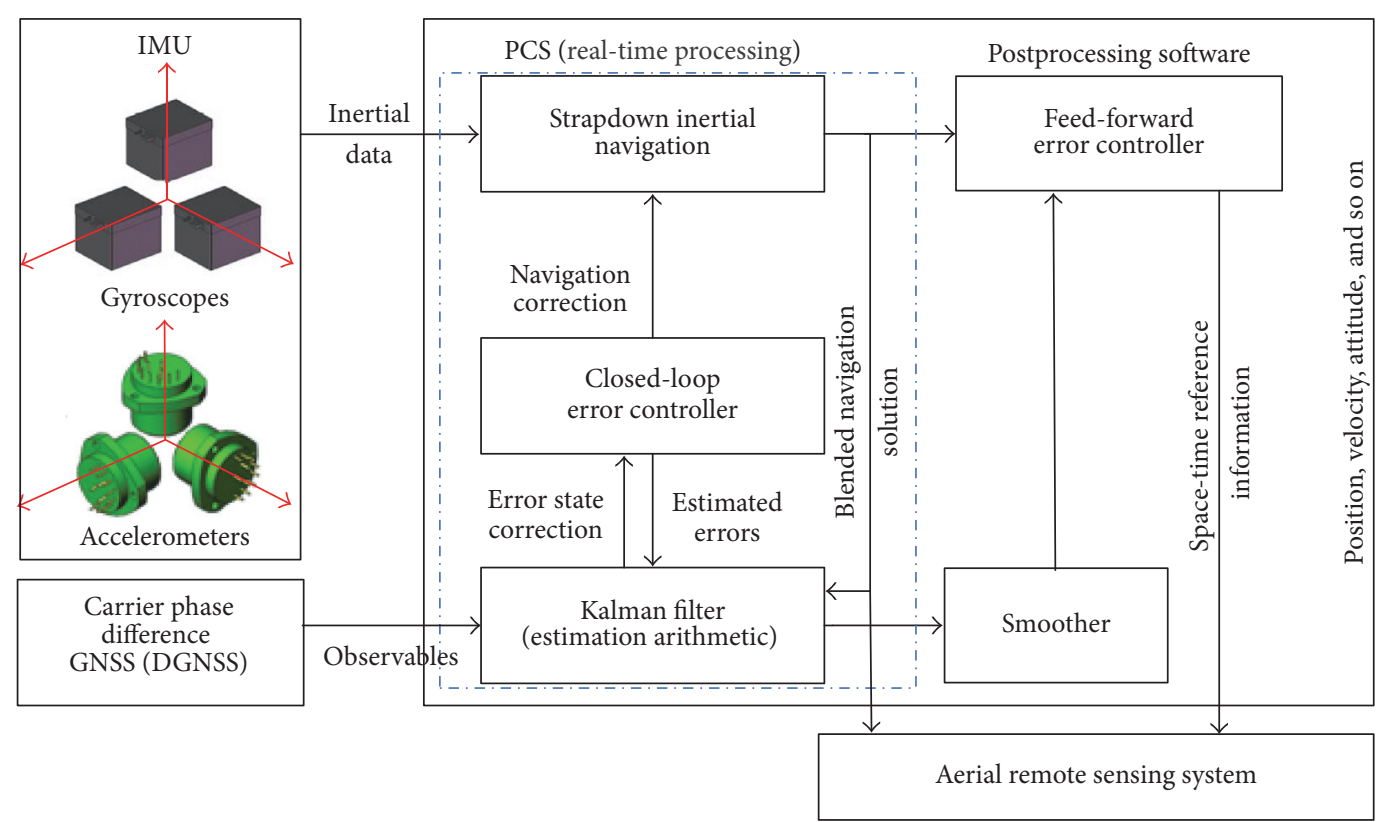

FIGURE 1: Operation principle and components of airborne POS.

noise or even outage. So, the inertial navigation system integrated with GNSS will produce an excellent solution that not only retains the dynamic capability of inertial navigation system, but also possesses the absolute position and velocity accuracy of the GNSS. Employing an integrate Kalman filter, the POS accurately measures the motion of the imaging sensor with high frequency and low noise solutions. This integrated arithmetic can be run in postprocess or real-time by PCS.

The inertial navigation solves velocity, position, and attitude of body by integrating angular rates and acceleration measured by IMU, based on Newton's laws. In order to do this, the inertial navigation system first needs to initialize velocity, position, and attitude of body. After removing the Earth rate, the navigator integrates the angular rate measured by gyros to continuously calculate the attitude of IMU with respect to local level navigation frame. Then, it computes the specific force measured by accelerometer to relative motion acceleration in navigation frame, first integrates to velocity, and second integrates to position of IMU. The dynamical solutions are very accurate [11]. However, the errors of velocity, position, and attitude solved by inertial navigation will slowly grow with time because of integration process. The IMU used in POS must be as lightweight and small as possible. It may be directly mounted onto or close to existing imaging sensors [12]. There is no flexible deformation between the imaging sensor and IMU, thus meaning that the inertial navigator solves velocity, position, and attitude of the imaging sensor itself. Currently, typical IMUs use either Fiber Optic Gyros (FOG) or Ring Laser Gyros (RLG). Every gyro has its advantage and technology shortage, and every technology mean can be applied to solve different problem.

The GNSS system solves position of the receiver's antenna using triangulation techniques and may operate in two different methods. The first one is single point positioning technology which uses a single receiver and is the most simple but loses the position precision. Its typical precision varies from $2 \mathrm{~m}$ to $10 \mathrm{~m}$ and is not suitable for most of aerial remote sensing system [13]. The second one is referred to as carrier phase differential GNSS which combines the phase from two receivers so as to eliminate clock errors, multipath effect, and so on. In most applications, the airborne POS uses an embedded dual frequency (L1/L2) GNSS receiver that operates in Real-Time Kinematics (RTK) or in postprocess. It need set up a dedicated reference GNSS receiver with a radio link to the roving GNSS receiver. The typical RTK accuracy is $1 \mathrm{~cm}$ plus $1 \mathrm{ppm}$ of baseline separation within $100 \mathrm{~km} \mathrm{[14].}$

As computing and processing heart of POS, the PCS mainly consists of data processing module that runs data sampling and real-time integrated navigation algorithm; a roving GNSS receiver module that sometimes independently operates and provides velocity, position, and time reference information; a data storage module that writes data to a pluggable CF/SD card or flash disk; and a power module which provides various voltages for other modules in PCS and IMU. The PCS controls the data acquisition from GNSS and IMU and can store the data in storage modules. Using GNSS time and pulse per second (PPS), all acquired sensor data can be accurately timed with time-tags [15]. The data processing module can execute error compensation of IMU, initial alignment and inertial navigation algorithm, and realtime integrated filter as shown in Figure 1. For the sake of improving the real-time performance, the frequency of data processing module can reach as high as $500 \mathrm{~Hz}$. The PCS can communicate with external devices by some digital and analog input/output interfaces, RS232, RS422, LAN, $\mathrm{CAN}$, and so on. The POS can provide accurate space-time referencing information including velocity, position, attitude 
in real-time for the inertial stabilized platforms, imaging sensors, and flight management systems [16, 17].

The postprocessing software can calculate a superior solution by integrating raw reference and roving GNSS data from storage module. It computes a carrier phase differential GNSS solution and then integrates with the inertial data using forward and reverse in time processing $[18,19]$. The postprocessing algorithm can provide a dramatic improvement in accuracy, which is helpful for poor GNSS or GNSS outages. It mainly contains two parts: the smoother and the feedforward error controller as shown in Figure 1.

\section{Key Technologies of POS}

3.1. IMU Error Calibration and Compensation. The aim of IMU error calibration is to calculate the parameters of error model. Generally, the Earth rate $\boldsymbol{\omega}_{\text {ie }}$, gravity acceleration $\mathbf{g}$, and input angular rate of inertial turntable $\boldsymbol{\Omega}$ are regarded as references, which are compared with outputs of accelerometers and gyros of IMU. The parameters of IMU error model can be resolved by analytic methods. An accurate calibration and compensation method is necessary to advance the IMU measurement accuracy $[20,21]$.

3.1.1. IMU Error Modeling. The IMU is composed of three orthogonal gyros and accelerometers. Since the gravity acceleration can not cause IMU measurement error, considering the biases, scale factors, and misalignments, the error model of angular rate channel can be given by $[22,23]$

$$
\left[\begin{array}{c}
G_{x} \\
G_{y} \\
G_{z}
\end{array}\right]=\left[\begin{array}{c}
B_{g x} \\
B_{g y} \\
B_{g z}
\end{array}\right]+\left[\begin{array}{ccc}
K_{x} & E_{x y} & E_{x z} \\
E_{y x} & K_{y} & E_{y z} \\
E_{z x} & E_{z y} & K_{z}
\end{array}\right]\left[\begin{array}{c}
\omega_{x} \\
\omega_{y} \\
\omega_{z}
\end{array}\right]
$$

where $B_{g i}$ and $G_{i}$ are gyros bias and output along $i$ axis, $\omega_{i}$ is the rational rate input by inertial turntable, $K_{i}$ is gyros scale factor, and $E_{i j}$ is the misalignment of angular rate channel. $i=$ $x, y, z ; j=x, y, z ; i \neq j$. According to the quartz mechanical accelerometer characteristic, the IMU error model of acceleration channel may be expressed as

$$
\left[\begin{array}{c}
f_{x} \\
f_{y} \\
f_{z}
\end{array}\right]=\left[\begin{array}{c}
B_{a x} \\
B_{a y} \\
B_{a z}
\end{array}\right]+\left[\begin{array}{ccc}
S_{x} & H_{x y} & H_{x z} \\
H_{y x} & S_{y} & H_{y z} \\
H_{z x} & H_{z y} & S_{z}
\end{array}\right]\left[\begin{array}{c}
a_{x} \\
a_{y} \\
a_{z}
\end{array}\right]
$$

where $B_{a i}$ and $f_{i}$ are the accelerometer bias and specific force in $i$ axis, $a_{i}$ is the input acceleration, $S_{i}$ is the acceleration scale factor, and $H_{i j}$ is the misalignment of the acceleration channel, $i=x, y, z ; j=x, y, z ; i \neq j$.

3.1.2. Error Calibration of IMU. According to the IMU error model, a rotation calibration method with six directions is designed based on inertial turntable. The IMU can be overturned six times with the help of hexahedral fixture. Thus, the $x, y$, and $z$-axes of IMU are, respectively, superposed with $Z_{t}$ and $-Z_{t}$ axes of inertial turntable $\left(Z_{t}\right.$ axes in navigation frame). At each direction, the inertial turntable should be rotated $2 \pi n$ angle with $n$ being rotation periods. When $x$-axis points to first direction, the input angular rates of the three sensing axes can be written by

$$
\left[\begin{array}{c}
\omega_{x}(t) \\
\omega_{y}(t) \\
\omega_{z}(t)
\end{array}\right]=\left[\begin{array}{c}
\omega_{i e} \cos L \sin \phi(t) \\
\omega_{i e} \cos L \cos \phi(t) \\
\Omega+\omega_{i e} \sin L
\end{array}\right],
$$

where $L$ is local latitude. $\phi(t)$ is IMU head in $t$ moment. The input acceleration along three axes can be written by

$$
\left[\begin{array}{l}
a_{x}(t) \\
a_{y}(t) \\
a_{z}(t)
\end{array}\right]=\left[\begin{array}{l}
0 \\
0 \\
g
\end{array}\right]
$$

The means of input angular rates and acceleration at the first direction may be calculated as

$$
\begin{aligned}
& {\left[\begin{array}{l}
\omega_{x} \\
\omega_{y} \\
\omega_{z}
\end{array}\right]=\frac{\Omega}{2 \pi n}\left[\begin{array}{l}
\int_{0}^{2 \pi n / \Omega} \omega_{x}(t) d t \\
\int_{0}^{2 \pi n / \Omega} \omega_{y}(t) d t \\
\int_{0}^{2 \pi n / \Omega} \omega_{z}(t) d t
\end{array}\right]=\left[\begin{array}{c}
0 \\
0 \\
\Omega+\omega_{i e} \sin L
\end{array}\right]} \\
& =\left[\begin{array}{l}
0 \\
0 \\
\bar{\omega}
\end{array}\right], \\
& {\left[\begin{array}{l}
a_{x} \\
a_{y} \\
a_{z}
\end{array}\right]=\frac{\Omega}{2 \pi n}\left[\begin{array}{l}
\int_{0}^{2 \pi n / \Omega} a_{x}(t) d t \\
\int_{0}^{2 \pi n / \Omega} a_{y}(t) d t \\
\int_{0}^{2 \pi n / \Omega}(t) d t
\end{array}\right]=\left[\begin{array}{l}
0 \\
0 \\
g
\end{array}\right] .}
\end{aligned}
$$

According to (1) and (5), the system state equations of IMU angular rate channel can be written by

$$
\begin{aligned}
& {\left[\begin{array}{ccc}
G_{x 1} & G_{y 1} & G_{z 1} \\
G_{x 2} & G_{y 2} & G_{z 2} \\
G_{x 3} & G_{y 3} & G_{z 3} \\
G_{x 4} & G_{y 4} & G_{z 4} \\
G_{x 5} & G_{y 5} & G_{z 5} \\
G_{x 6} & G_{y 6} & G_{z 6}
\end{array}\right]} \\
& =\left[\begin{array}{cccc}
1 & \bar{\omega} & 0 & 0 \\
1 & -\bar{\omega} & 0 & 0 \\
1 & 0 & \bar{\omega} & 0 \\
1 & 0 & -\bar{\omega} & 0 \\
1 & 0 & 0 & \bar{\omega} \\
1 & 0 & 0 & -\bar{\omega}
\end{array}\right]\left[\begin{array}{lll}
B_{g x} & B_{g y} & B_{g z} \\
K_{x} & E_{y x} & E_{z x} \\
E_{x y} & K_{y} & E_{z y} \\
E_{x z} & E_{y z} & K_{z}
\end{array}\right],
\end{aligned}
$$

where $\bar{\omega}$ is input angular rate, $\bar{\omega}=\Omega+\omega_{i e} \sin L . G_{i m}$ is gyros output along $i$ axes for $m$ th direction, $i=x, y, z$; 
$m=1,2,3,4,5,6$. According to (2) and (6), the IMU acceleration channel can be written by

$$
\begin{aligned}
& {\left[\begin{array}{ccc}
f_{x 1} & f_{y 1} & f_{z 1} \\
f_{x 2} & f_{y 2} & f_{z 2} \\
f_{x 3} & f_{y 3} & f_{z 3} \\
f_{x 4} & f_{y 4} & f_{z 4} \\
f_{x 5} & f_{y 5} & f_{z 5} \\
f_{x 6} & f_{y 6} & f_{z 6}
\end{array}\right]} \\
& =\left[\begin{array}{cccc}
1 & g & 0 & 0 \\
1 & -g & 0 & 0 \\
1 & 0 & g & 0 \\
1 & 0 & -g & 0 \\
1 & 0 & 0 & g \\
1 & 0 & 0 & -g
\end{array}\right]\left[\begin{array}{ccc}
B_{a x} & B_{a y} & B_{a z} \\
S_{x} & H_{y x} & H_{z x} \\
H_{x y} & S_{y} & H_{z y} \\
H_{x z} & H_{y z} & S_{z}
\end{array}\right],
\end{aligned}
$$

where $f_{i m}$ is the specific force measured by $i$ axes accelerometer for $m$ th direction. According to (7) and (8), the IMU error parameters may be solved as

$$
\begin{aligned}
& {\left[\begin{array}{lll}
B_{g x} & B_{g y} & B_{g z} \\
K_{x} & E_{y x} & E_{z x} \\
E_{x y} & K_{y} & E_{z y} \\
E_{x z} & E_{y z} & K_{z}
\end{array}\right]} \\
& =\frac{1}{2 \bar{\omega}}\left[\begin{array}{ccc}
\frac{\bar{\omega}}{3} \sum_{i=1}^{6} G_{x i} & \frac{\bar{\omega}}{3} \sum_{i=1}^{6} G_{y i} & \frac{\bar{\omega}}{3} \sum_{i=1}^{6} G_{z i} \\
G_{x 1}-G_{x 2} & G_{y 1}-G_{y 2} & G_{z 1}-G_{z 2} \\
G_{x 3}-G_{x 4} & G_{y 3}-G_{y 4} & G_{z 3}-G_{z 4} \\
G_{x 5}-G_{x 6} & G_{y 5}-G_{y 6} & G_{z 5}-G_{z 6}
\end{array}\right], \\
& {\left[\begin{array}{ccc}
B_{a x} & B_{a y} & B_{a z} \\
S_{x} & H_{y x} & H_{z x} \\
H_{x y} & S_{y} & H_{z y} \\
H_{x z} & H_{y z} & S_{z}
\end{array}\right]} \\
& =\frac{1}{2 g}\left[\begin{array}{ccc}
\frac{g}{3} \sum_{i=1}^{6} A_{x i} & \frac{g}{3} \sum_{i=1}^{6} A_{y i} & \frac{g}{3} \sum_{i=1}^{6} A_{z i} \\
f_{x 1}-f_{x 2} & f_{y 1}-f_{y 2} & f_{z 1}-f_{z 2} \\
f_{x 3}-f_{x 4} & f_{y 3}-f_{y 4} & f_{z 3}-f_{z 4} \\
f_{x 5}-f_{x 6} & f_{y 5}-f_{y 6} & f_{z 5}-f_{z 6}
\end{array}\right] .
\end{aligned}
$$

3.2. Initial Alignment for Airborne POS. The motive of initial alignment is to calculate the initial attitude matrix $C_{n}^{b}$ of body. Two crucial destinations of initial alignment are how to improve accuracy and reduce time. The alignment accuracy decides measurement precision, and time reflects response performance of SINS $[24,25]$. Generally, the analytic coarse alignment based on stationary base is one of the most important initial alignment methods [26]. It is a self-alignment method independent of other sensors. It is well known that the Earth rate $\boldsymbol{\omega}_{\mathbf{i e}}$ and local gravity vector $\mathbf{g}$ could be sensed by gyros and accelerometers of IMU in the body frame. Therefore, the analytic coarse self-alignment can calculate the initial attitude matrix by measuring Earth rate and local gravity noncollinear vectors in two frames. The local level frame is selected as the navigation frame where the $x, y$, and $z$ represent the east, north, and upward vertical axis, respectively. The Earth rate and local gravity may be written in navigation frame:

$$
\begin{aligned}
& \mathbf{g}^{\mathbf{n}}=\left[\begin{array}{lll}
0 & 0 & -g
\end{array}\right]^{T}, \\
& \boldsymbol{\omega}_{\mathbf{i e}}^{\mathbf{n}}=\left[\begin{array}{lll}
0 & \omega_{i e} \cos (L) & \omega_{i e} \sin (L)
\end{array}\right]^{T},
\end{aligned}
$$

where $g$ and $\omega_{i e}$ are the values of local gravity and Earth rate. On the other hand, the Earth rate and local gravity in body frame can be written by

$$
\begin{gathered}
\mathbf{g}^{\mathbf{b}}=C_{n}^{b} \mathbf{g}^{\mathbf{n}}, \\
\boldsymbol{\omega}_{\mathrm{ie}}^{\mathbf{b}}=C_{n}^{b} \boldsymbol{\omega}_{\mathrm{ie}}^{\mathbf{n}} .
\end{gathered}
$$

Therefore, the airborne POS outputs in angular rate and acceleration channels can be, respectively, expressed as

$$
\begin{aligned}
& {\left[\begin{array}{c}
f_{x} \\
f_{y} \\
f_{z}
\end{array}\right]=\left[\begin{array}{lll}
T_{11} & T_{12} & T_{13} \\
T_{21} & T_{22} & T_{23} \\
T_{31} & T_{32} & T_{33}
\end{array}\right]\left[\begin{array}{c}
0 \\
0 \\
-g
\end{array}\right],} \\
& {\left[\begin{array}{l}
G_{x} \\
G_{y} \\
G_{z}
\end{array}\right]=\left[\begin{array}{lll}
T_{11} & T_{12} & T_{13} \\
T_{21} & T_{22} & T_{23} \\
T_{31} & T_{32} & T_{33}
\end{array}\right]\left[\begin{array}{c}
0 \\
\omega_{i e} \cos (L) \\
\omega_{i e} \sin (L)
\end{array}\right],}
\end{aligned}
$$

where $T_{i j}$ is an element of attitude matrix from navigation frame to body frame $C_{n}^{b}, i, j=1,2,3$. The partial elements of matrix can be calculated by

$$
\begin{aligned}
& {\left[\begin{array}{c}
T_{13} \\
T_{23} \\
T_{33}
\end{array}\right]=-\frac{1}{g}\left[\begin{array}{l}
f_{x} \\
f_{y} \\
f_{z}
\end{array}\right],} \\
& {\left[\begin{array}{c}
T_{12} \\
T_{22} \\
T_{32}
\end{array}\right]=\frac{1}{\omega_{i e} \cos (L)}\left[\begin{array}{l}
G_{x} \\
G_{y} \\
G_{z}
\end{array}\right]+\frac{\tan (L)}{g}\left[\begin{array}{c}
f_{x} \\
f_{y} \\
f_{z}
\end{array}\right] .}
\end{aligned}
$$


TABLE 1: The roll angles of body.

\begin{tabular}{lccc}
\hline$\left\|T_{33}\right\|$ & $T_{33}$ & $T_{13}$ & $\gamma$ \\
\hline \multirow{2}{*}{$\geq e$} & $<0$ & $>0$ & $\gamma-\pi$ \\
& & $\leq 0$ & $\gamma+\pi$ \\
& $\geq 0$ & & $\gamma$ \\
$<e$ & & $>0$ & $-\pi / 2$ \\
& & $\leq 0$ & $\pi / 2$ \\
\hline
\end{tabular}

TABLE 2: The head angles of body.

\begin{tabular}{lccc}
\hline$\left\|T_{22}\right\|$ & $T_{22}$ & $T_{21}$ & $\phi$ \\
\hline \multirow{2}{*}{$\geq e$} & $>0$ & $>0$ & $\phi+2 \pi$ \\
& & $\leq 0$ & $\phi$ \\
\hline$<e$ & & & $\phi+\pi$ \\
\hline & & $>0$ & $3 \pi / 2$ \\
& & $\leq 0$ & $\pi / 2$ \\
\hline
\end{tabular}

According the matrix orthogonality, the remnant elements may be calculated. Therefore, the body attitude matrix may be given by

$$
\begin{aligned}
C_{n}^{b} & =\left[\begin{array}{ccc}
T_{11} & T_{12} & T_{13} \\
T_{21} & T_{22} & T_{23} \\
T_{31} & T_{32} & T_{33}
\end{array}\right]=\left[\begin{array}{lll}
T_{22} T_{33}-T_{32} T_{23} & T_{12} & T_{13} \\
T_{13} T_{32}-T_{12} T_{33} & T_{22} & T_{23} \\
T_{12} T_{23}-T_{13} T_{22} & T_{32} & T_{33}
\end{array}\right] \\
& =\left[\begin{array}{ccc}
\frac{f_{y} G_{z}-f_{z} G_{y}}{g \Omega \cos (L)} & \frac{G_{x}}{\Omega \cos (L)}+\frac{f_{x}}{g} \tan (L) & -\frac{f_{x}}{g} \\
\frac{f_{z} G_{x}-f_{x} G_{z}}{g \Omega \cos (L)} & \frac{G_{y}}{\Omega \cos (L)}+\frac{f_{y}}{g} \tan (L) & -\frac{f_{y}}{g} \\
\frac{f_{x} G_{y}-f_{y} G_{x}}{g \Omega \cos (L)} & \frac{G_{z}}{\Omega \cos (L)}+\frac{f_{z}}{g} \tan (L) & -\frac{f_{z}}{g}
\end{array}\right] .
\end{aligned}
$$

According to (14), the initial attitude angles of body may be obtained as [27]

$$
\begin{aligned}
& \theta=\sin ^{-1}\left(T_{23}\right)=\sin ^{-1}\left(-\frac{f_{y}}{g}\right), \\
& \gamma=\tan ^{-1}\left(-\frac{T_{13}}{T_{33}}\right)=\tan ^{-1}\left(-\frac{f_{x}}{f_{z}}\right), \\
& \phi=\tan ^{-1}\left(-\frac{T_{21}}{T_{22}}\right)=\tan ^{-1}\left(\frac{f_{x} G_{z}-f_{z} G_{x}}{g G_{y}+f_{y} \Omega \sin (L)}\right) .
\end{aligned}
$$

By identifying partial elements signs of attitude matrix, the roll and head angles of body can be calculated as in Tables 1 and 2 (Note: $e=1 \times 10^{-12}$ ).

3.3. Lever Arm Error Modeling and Compensation. The lever arm denotes a spatial difference vector between two frames originating from different sensors. It makes the sensed motion information different from the others, which include position and velocity. The motion error is affected by angular velocity and lever arm length and so on. Airborne POS

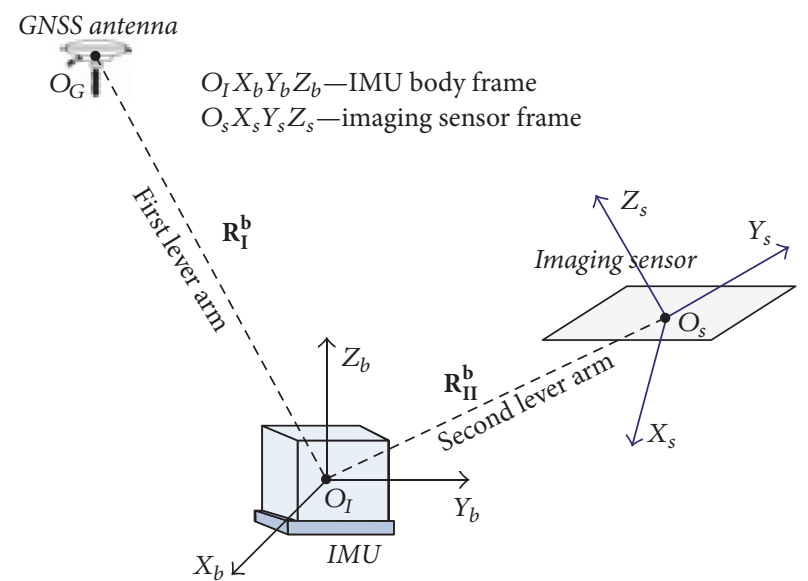

Figure 2: Multilevel rigid lever arm mechanism.

applied in aerial remote sensing system contains three types of sensor, GNSS, IMU, and imaging sensor with some lever arms among them. It can be called multilevel rigid lever arm, generally [28].

The multilevel rigid lever arm mechanism is shown in Figure 2. When the GNSS antenna is installed in top of airplane, there exists a lever arm towards the IMU. The first lever arm $\mathbf{R}_{\mathbf{I}}^{\mathbf{b}}$ describes the spatial difference vector between measurement centers of IMU and GNSS antenna. The second lever arm, $\mathbf{R}_{\mathrm{II}}^{\mathbf{b}}$, denotes the spatial difference vector between sensing centers of IMU and imaging sensor. The motion of IMU may be precisely solved by first lever arm compensation. However, only when the second lever arm is compensated, can the motion solution transfer into the sensing center of imaging sensor $[29,30]$.

3.3.1. First Lever Arm Error Modeling and Compensation. The spatial difference vector caused by first lever arm may be written by

$$
\Delta \mathbf{P}_{\mathbf{I}}^{\mathbf{n}}=C_{b}^{n} \mathbf{R}_{\mathbf{I}}^{\mathbf{b}}
$$

where $C_{b}^{n}$ is the body attitude matrix, $C_{b}^{n}=\left[C_{n}^{b}\right]^{-1}=\left[C_{n}^{b}\right]^{T}$, that can be obtained as (15); $T_{i j}$ represents an element of attitude matrix $C_{n}^{b}$ with $i, j=1,2,3 . \mathbf{R}_{\mathbf{I}}^{\mathbf{b}}$ represents a first lever arm vector, $\phi, \theta$, and $\gamma$ represent body head, pitch, and roll angles. Using the first lever arm compensation method, the position measured by GNSS $\mathbf{P}_{\mathbf{G}}^{\mathbf{n}}$ can be converted to sensing center of IMU that is expressed as

$$
\begin{aligned}
& \mathbf{P}_{\mathbf{I G}}^{\mathbf{n}}=\mathbf{P}_{\mathbf{G}}^{\mathbf{n}}+\Pi \Delta \mathbf{P}_{\mathbf{I}}^{\mathbf{n}}=\mathbf{P}_{\mathbf{I G}}^{\mathbf{n}}+\boldsymbol{\Pi}\left[C_{n}^{b}\right]^{T} \mathbf{R}_{\mathbf{I}}^{\mathbf{b}}, \\
& {\left[\begin{array}{c}
L_{I G} \\
\lambda_{I G} \\
H_{I G}
\end{array}\right]=\left[\begin{array}{c}
L_{G} \\
\lambda_{G} \\
H_{G}
\end{array}\right]} \\
& +\left[\begin{array}{ccc}
\frac{1}{\left(R_{M}+H\right)} & 0 & 0 \\
0 & \frac{\sec (L)}{\left(R_{N}+H\right)} & 0 \\
0 & 0 & 1
\end{array}\right]\left[\begin{array}{lll}
T_{11} & T_{21} & T_{31} \\
T_{12} & T_{22} & T_{32} \\
T_{13} & T_{23} & T_{33}
\end{array}\right]\left[\begin{array}{c}
X_{I} \\
Y_{I} \\
Z_{I}
\end{array}\right],
\end{aligned}
$$


where $L_{G}, \lambda_{G}$, and $H_{G}$ represent latitude, longitude, and altitude measured by GNSS, respectively, $L$ and $H$ are latitude and altitude measured by POS in last time, $R_{I}^{b}=$ $\left[\begin{array}{lll}X_{I} & Y_{I} & Z_{I}\end{array}\right]^{T}$ represent the lengths of first lever arm in body frame, and $R_{M}$ and $R_{N}$ are the transverse and meridian radii of curvature and can be written as

$$
\begin{aligned}
& R_{M}=\frac{R_{0}\left(1-e^{2}\right)}{\left(1-e^{2} \sin ^{2} L\right)^{3 / 2}}, \\
& R_{N}=\frac{R_{0}}{\left(1-e^{2} \sin ^{2} L\right)^{1 / 2}},
\end{aligned}
$$

where $R_{0}$ is the Earth radius; $e$ is the flat ratio. The velocity error vector induced by lever arm in navigation frame can be written by

$$
\Delta \mathbf{V}_{\mathbf{I}}^{\mathbf{n}}=C_{b}^{n}\left(\boldsymbol{\omega}_{\mathbf{n b}}^{\mathbf{b}} \times \mathbf{R}_{\mathbf{I}}^{\mathbf{b}}\right)
$$

where $\boldsymbol{\omega}_{\mathbf{n} \mathbf{b}}^{\mathbf{b}}$ is an angular rate vector of body with respect to navigation coordinate frame expressed in body coordinate frame and may be calculated:

$$
\begin{aligned}
& \omega_{\mathrm{nb}}^{\mathrm{b}}=\omega_{\mathrm{ib}}^{\mathrm{b}}-\omega_{\mathrm{in}}^{\mathrm{b}}=\omega_{\mathrm{ib}}^{\mathrm{b}}-C_{n}^{b} \omega_{\mathrm{in}}^{\mathrm{n}} \\
& =\boldsymbol{\omega}_{\mathrm{ib}}^{\mathbf{b}}-C_{n}^{b}\left(\boldsymbol{\omega}_{\mathrm{ie}}^{\mathbf{n}}+\boldsymbol{\omega}_{\mathrm{en}}^{\mathbf{n}}\right), \\
& {\left[\begin{array}{c}
\omega_{n b x}^{b} \\
\omega_{n b y}^{b} \\
\omega_{n b z}^{b}
\end{array}\right]} \\
& =\left[\begin{array}{l}
\omega_{i b x}^{b} \\
\omega_{i b y}^{b} \\
\omega_{i b z}^{b}
\end{array}\right] \\
& -\left[\begin{array}{lll}
T_{11} & T_{12} & T_{13} \\
T_{21} & T_{22} & T_{23} \\
T_{31} & T_{32} & T_{33}
\end{array}\right]\left[\begin{array}{c}
-\frac{V_{N}}{R_{M}} \\
\omega_{i e} \cos (L)+\frac{V_{E}}{R_{N}} \\
\omega_{i e} \sin (L)+\frac{V_{E} \tan (L)}{R_{N}}
\end{array}\right],
\end{aligned}
$$

where $\omega_{\mathrm{ib}}^{\mathbf{b}}$ is the angular rate vector of body coordinate frame with respect to inertial coordinate frame expressed in body coordinate frame, which can be directly sensed by gyros. $V_{E}, V_{N}$, and $V_{U}$, respectively, represent velocities along east, north, and upward axis at previous moment. Using the compensation arithmetic of first lever arm, the velocity $\mathbf{V}_{\mathbf{G}}^{\mathbf{n}}$ sensed by GNSS antenna can be converted to sensing center of IMU that is expressed as

$$
\mathbf{V}_{\mathbf{I G}}^{\mathbf{n}}=\mathbf{V}_{\mathbf{G}}^{\mathbf{n}}-\Delta \mathbf{V}_{\mathbf{I}}^{\mathbf{n}}=\mathbf{V}_{\mathbf{G}}^{\mathbf{n}}-C_{b}^{n}\left(\boldsymbol{\omega}_{\mathbf{n b}}^{\mathbf{b}} \times \mathbf{R}_{\mathbf{I}}^{\mathbf{b}}\right),
$$

$$
\begin{gathered}
{\left[\begin{array}{c}
V_{I G E}^{n} \\
V_{I G N}^{n} \\
V_{I G U}^{n}
\end{array}\right]=\left[\begin{array}{c}
V_{G E}^{n} \\
V_{G N}^{n} \\
V_{G U}^{n}
\end{array}\right]} \\
-\left[\begin{array}{lll}
T_{11} & T_{21} & T_{31} \\
T_{12} & T_{22} & T_{32} \\
T_{13} & T_{23} & T_{33}
\end{array}\right]\left[\begin{array}{ccc}
0 & -\omega_{n b z}^{b} & \omega_{n b y}^{b} \\
\omega_{n b z}^{b} & 0 & -\omega_{n b x}^{b} \\
-\omega_{n b y}^{b} & \omega_{n b x}^{b} & 0
\end{array}\right]\left[\begin{array}{c}
X_{I} \\
Y_{I} \\
Z_{I}
\end{array}\right] .
\end{gathered}
$$

3.3.2. Second Lever Arm Error Modeling and Compensation. The second lever arm error compensation refers to transfer POS measurement solutions to the sensing center of imaging sensor. The latitude, longitude, and altitude compensated by second lever arm can be written by

$$
\begin{aligned}
& \mathbf{P}_{\mathbf{S P}}^{\mathbf{n}}=\mathbf{P}_{\mathbf{P}}^{\mathbf{n}}+\Pi \Delta \mathbf{P}_{\mathrm{II}}^{\mathbf{n}}=\mathbf{P}_{\mathbf{P}}^{\mathbf{n}}+\Pi\left[C_{n}^{b}\right]^{T} \mathbf{R}_{\mathrm{II}}^{\mathbf{b}}, \\
& {\left[\begin{array}{c}
L_{S P} \\
\lambda_{S P} \\
H_{S P}
\end{array}\right]=\left[\begin{array}{c}
L_{P} \\
\lambda_{P} \\
H_{P}
\end{array}\right]} \\
& \quad+\left[\begin{array}{ccc}
\frac{1}{\left(R_{M}+H\right)} & 0 & 0 \\
0 & \frac{\sec (L)}{\left(R_{N}+H\right)} & 0 \\
0 & 0 & 1
\end{array}\right]\left[\begin{array}{lll}
T_{11} & T_{21} & T_{31} \\
T_{12} & T_{22} & T_{32} \\
T_{13} & T_{23} & T_{33}
\end{array}\right]\left[\begin{array}{c}
X_{I I} \\
Y_{I I} \\
Z_{I I}
\end{array}\right],
\end{aligned}
$$

where $\mathbf{P}_{\mathbf{P}}^{\mathbf{n}}$ is position vector solved by POS; $\Delta \mathbf{P}_{\mathrm{II}}^{\mathbf{n}}$ represents displacement vector caused by the second lever arm $\mathbf{R}_{\mathrm{II}}^{\mathbf{b}} \cdot L_{P}$, $\lambda_{P}$, and $H_{P}$ are latitude, longitude, and altitude measured by POS, respectively. Based on velocity vector $\mathbf{V}_{\mathbf{P}}^{\mathbf{n}}$ solved by POS, the velocity compensated for the sensing center of imaging sensor by second lever arm can be written as

$$
\begin{aligned}
& \mathbf{V}_{\mathbf{S P}}^{\mathbf{n}}=\mathbf{V}_{\mathbf{P}}^{\mathbf{n}}+\Delta \mathbf{V}_{\mathbf{I I}}^{\mathbf{n}}=\mathbf{V}_{\mathbf{P}}^{\mathbf{n}}+C_{b}^{n}\left(\boldsymbol{\omega}_{\mathbf{n b}}^{\mathbf{b}} \times \mathbf{R}_{\mathrm{II}}^{\mathbf{b}}\right), \\
& {\left[\begin{array}{c}
V_{S P E}^{n} \\
V_{S P N}^{n} \\
V_{S P U}^{n}
\end{array}\right]=\left[\begin{array}{c}
V_{P E}^{n} \\
V_{P N}^{n} \\
V_{P U}^{n}
\end{array}\right]} \\
& +\left[\begin{array}{lll}
T_{11} & T_{21} & T_{31} \\
T_{12} & T_{22} & T_{32} \\
T_{13} & T_{23} & T_{33}
\end{array}\right]\left[\begin{array}{ccc}
0 & -\omega_{n b z}^{b} & \omega_{n b y}^{b} \\
\omega_{n b z}^{b} & 0 & -\omega_{n b x}^{b} \\
-\omega_{n b y}^{b} & \omega_{n b x}^{b} & 0
\end{array}\right]\left[\begin{array}{c}
X_{I I} \\
Y_{I I} \\
Z_{I I}
\end{array}\right],
\end{aligned}
$$

where $\Delta \mathbf{V}_{\text {II }}^{\mathbf{n}}$ represents velocity error vector caused by the second lever arm.

3.4. Time Synchronization of Multisensors. Time synchronization is very important to airborne POS. As shown in Figure 3, the three sensors, GNSS, SINS, and imaging sensor, respectively, work with their time sequence. Because time synchronization plays an important role in achieving excellent information fusion capability, these data measured by different sensors must to be accurately synchronized before fusing them together. The time synchronization technology of multisensors is critical for ensuring measurement accuracy 


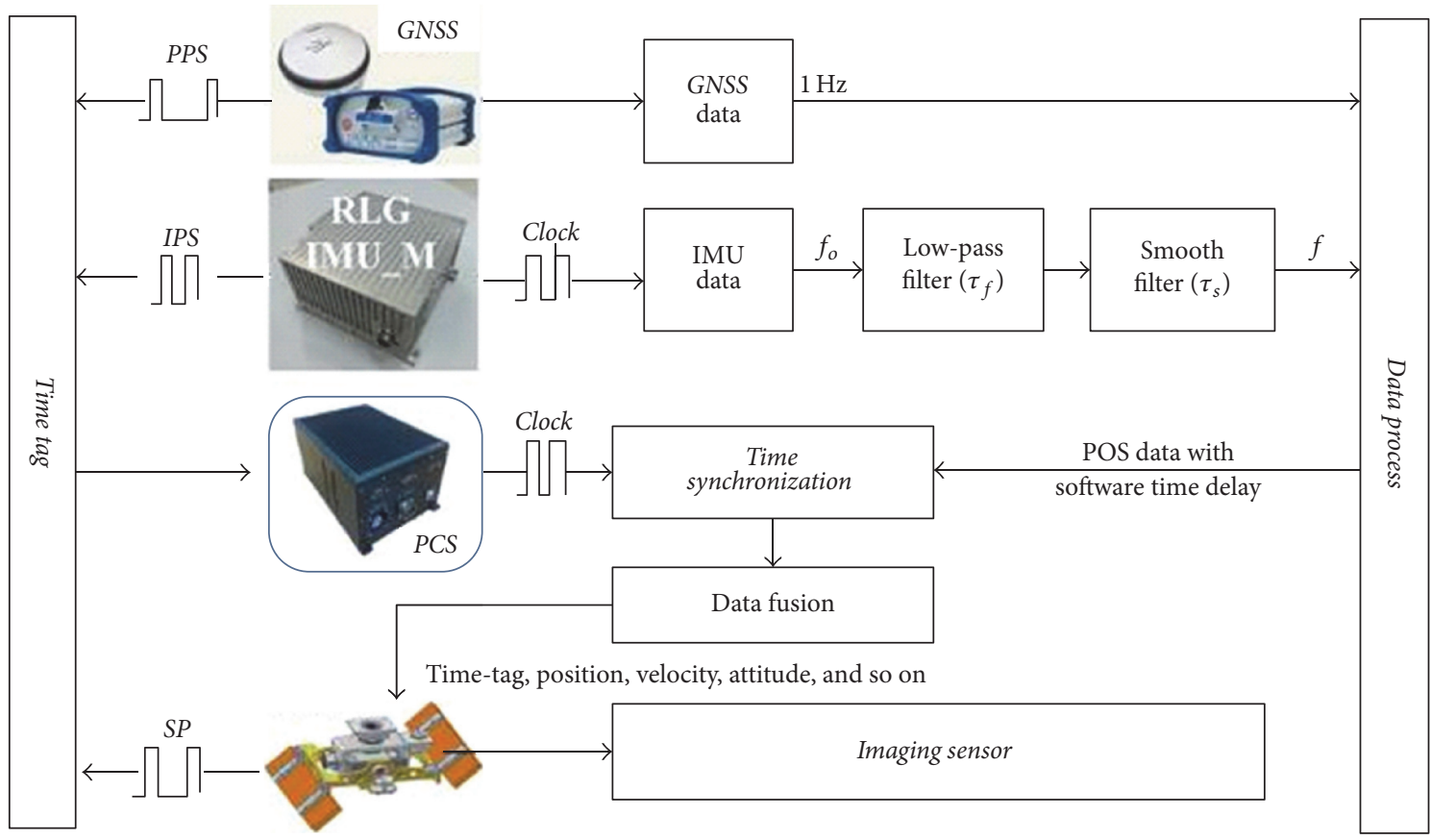

FIGURE 3: Timing and synchronization principle of POS.

of POS and then advances the imaging performance of aerial remote sensing system.

The GNSS can output a high precision time reference which is Universal Time Coordinated (UTC), while emitting a pulse per second (PPS). Generally, both INS and imaging sensor do not show special timing module and can not directly synchronize to time reference provided by GNSS receiver. On the other hand, some image sensors rarely collect data with a fixed frequency. Furthermore, the synchronization error also comes from algorithm time delay of software and crystals oscillator drift of hardware. These time synchronization errors are complicated and multisource and must to be solved, respectively, by various technologies and methods. Thereinto, the crystals oscillator drift of hardware may be calibrated using UTC time and PPS of GNSS receiver and then accurately compensated for UTC. Therefore, the airborne POS solutions will be timed with a time-tag synchronizing to GNSS, so as to be used for a direct georeference or motion compensation system of aerial remote sensing system [31]. However, these algorithm time delays of software are intractable problems and barely mentioned. In this paper, the first thing is to introduce time delays caused by filter and smooth algorithm.

In order to eliminate dynamic lock-in error, a mechanically dithered technique is adopted in RLG which high accuracy angular rate sensor. However, adverse noise appears in the RLG data and will cause measurement noise and error of airborne POS. A digital finite impulse response (FIR) filter is an effective algorithm to eliminate fearful data noise. It has been applied to RLG POS but results in time delay and can be written by $[32,33]$

$$
\tau_{F}=\frac{N-1}{2 f_{o}},
$$

where $N$ represents filter order; $T^{\prime}$ represents sampling period $T^{\prime}=1 / f_{o}$ with $f_{o}$ being frequency of RLG.

A smooth filter is also used in RLG noise attenuation. It will divide data sampling frequency $f_{o}$ into $f$ groups and then outputs mean value of every group of data. Using these data, navigator can compute velocity, position, and attitude solutions, since the operation principle of smooth filter is similar to that of FIR filter mentioned above and also brings about data time delay. If smooth filter order $N^{\prime}$ is equivalent to $f_{o} / f$, the time delay induced by smooth filter may be written by

$$
\tau_{S}=\frac{N^{\prime}-1}{2 f_{o}}=\frac{f_{o}-f}{2 f_{o} f} .
$$

3.5. Integrated Estimation of Postprocessing Software. Kalman filter is the most practical integrated navigation algorithm which can be used to fuse INS and GNSS data and produce an excellent measurement solution $[34,35]$. Besides, a postprocessing integrated estimation method has become one of the key technologies of airborne POS. Since the smoother uses more observations, the postprocessing method can further advance measurement accuracy. Nowadays, there are three kinds of smoothers which are fixed interval smoother, the fixed point smoother, and the fixed lag smoother. Thereinto, an Extended Rauch-Tung-Striebel fixed interval smoother (ERTSS) is applied in airborne POS due to its effectiveness and robustness. It may be decomposed into two parts that are called forward filtering process and the backward recursion process. After filtering in the forward process, the smoothing algorithm in backward process is used to compute smoothing solutions [36]. Thereinto, the forward process 

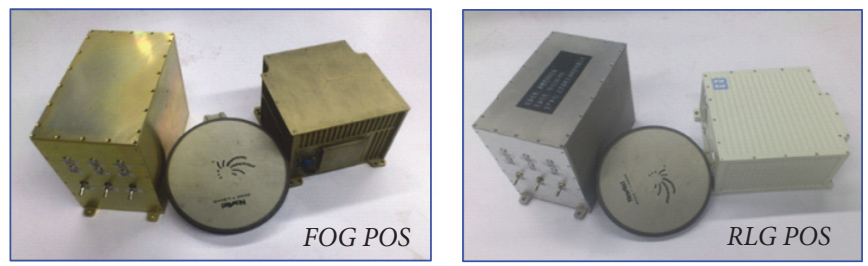

Figure 4: Airborne POS developed by BUAA.

adopts Extended Kalman Filter (EKF) which can be expressed as

$$
\begin{aligned}
\widehat{\mathbf{x}}_{k / k-1} & =\boldsymbol{\Phi}_{k / k-1} \widehat{\mathbf{x}}_{k-1}, \\
\mathbf{P}_{k / k-1} & =\boldsymbol{\Phi}_{k / k-1} \mathbf{P}_{k-1} \boldsymbol{\Phi}_{k / k-1}^{T}+\mathbf{G}_{k-1} \mathbf{Q}_{k-1} \mathbf{G}_{k-1}{ }^{T}, \\
\mathbf{K}_{k} & =\mathbf{P}_{k / k-1} \mathbf{H}_{k}^{T}\left(\mathbf{H}_{k} \mathbf{P}_{k / k-1} \mathbf{H}_{k}^{T}+\mathbf{R}_{k}\right)^{-1}, \\
\widehat{\mathbf{x}}_{k} & =\widehat{\mathbf{x}}_{k / k-1}+\mathbf{K}_{k}\left(\mathbf{z}_{k}-\mathbf{H}_{k} \widehat{\mathbf{x}}_{k / k-1}\right), \\
\mathbf{P}_{k} & =\left(\mathbf{I}-\mathbf{K}_{k} \mathbf{H}_{k}\right) \mathbf{P}_{k / k-1}\left(\mathbf{I}-\mathbf{K}_{k} \mathbf{H}_{k}\right)^{T}+\mathbf{K}_{k} \mathbf{R}_{k} \mathbf{K}_{k}^{T},
\end{aligned}
$$

where $\widehat{\mathbf{x}}_{k}$ and $\widehat{\mathbf{x}}_{k / k-1}$ represent posterior and prior estimates of state $\mathbf{x}_{k}$, respectively, $\mathbf{P}_{k}$ and $\mathbf{P}_{k / k-1}$ are error covariance matrix of state $\mathbf{x}_{k}$, respectively, and $\mathbf{K}_{k}$ is the gain matrix of EKF. The backward recursion process may adopt R-T-S algorithm which can be expressed as [36]

$$
\begin{aligned}
& \mathbf{K}_{k}^{S}=\mathbf{P}_{k} \boldsymbol{\Phi}_{k / k-1}^{T}\left(\mathbf{P}_{k / k+1}\right)^{-1}, \\
& \widehat{\mathbf{x}}_{k}^{S}=\widehat{\mathbf{x}}_{k}+\mathbf{K}_{k}^{S}\left(\widehat{\mathbf{x}}_{k+1}^{S}-\widehat{\mathbf{x}}_{k / k+1}\right), \\
& \mathbf{P}_{k}^{S}=\mathbf{P}_{k}+\mathbf{K}_{k}^{S}\left(\mathbf{P}_{k+1}^{S}-\mathbf{P}_{k / k+1}\right)\left(\mathbf{K}_{k}^{S}\right)^{T},
\end{aligned}
$$

where the subscript $k$ represents the step number of filter, $k=N-1, N-2, \ldots, 0$, with $N$ being the last step, and the superscript $S$ refers to smoothing algorithm.

\section{Development and Application Experiment}

The airborne POS theory was researched since 1999 in BUAA University. Presently, high precision airborne RLG and FOG POS had been developed as shown in Figure 4. The airborne POS consists of IMU, PCS integrated with GNSS receiver, and postprocessing software. Different IMU types based on FOG or RLG technology can be provided with different accuracies to satisfy the user's requirements. The high precision FOG POS and RLG POS have been widely applied in InSAR, LIDAR, hyperspectral scanners, and digital cameras and advance imaging precision and efficiency, evidently.

4.1. Application Experiment in Airborne Optical Camera. As shown in Figure 5, the developed RLG POS had been applied in optical camera of aerial remote sensing system. It dynamically measured position and attitude of the camera and provided a georeference for optical camera. A flight experiment had been implemented, which consisted of a small airplane (A2C), integrated optical camera, optical camera unit, inertial stabilization platform, and the RLG POS. The RLG POS is fixed onto optical camera unit which is installed in the inertial stabilization platform. Using the solutions measured by RLG POS, the inertial stabilization platform mounted in airplane can control the optical camera unit to be local level navigation frame.

A large amount of Ground Control Points (GCP) was set up in a calibration field. Using triangulation techniques, the position and attitude of camera can be obtained accurately by these GCP at the exact moment of data capture for optical camera and may be regarded as a reference, then, to test and verify the accuracy of RLG POS. In this experiment, the attitude solutions of RLG POS were compared with camera attitudes reference calculated by GCP of 135 maps. The results show that the attitudes errors measured by RLG POS were advanced to $0.0028^{\circ}, 0.0043^{\circ}$, and $0.0020^{\circ}$. Using the developed RLG POS, the optical camera successfully achieved 1:500 scale maps without Ground Control Points.

4.2. Application Experiment in Airborne InSAR. The developed RLG POS had been applied in motion compensation of high-resolution $\mathrm{X}$ wave band InSAR as shown in Figure 6. On account of the performance of the dual-antenna InSAR, the developed RLG POS may achieve position error compensation, interferometer phase error compensation, and automatic registration of the InSAR image-pair during the imaging process. The RLG POS provide the ability to realize high quality imaging of airborne InSAR with $0.15 \mathrm{~m}$ horizontal and $0.5 \mathrm{~m}$ highness resolution. The results showed that the developed RLG POS contributed to motion imaging compensation of a dual-antenna airborne InSAR system. It observably advanced the image quality and the image-pair coherence.

4.3. Application Experiment in Airborne TSMFTIS. As shown in Figure 7, an airborne application experiment was implemented and included an experimental airplane (Y-12), Temporally and Spatially Modulated Fourier Transform Imaging Spectrometer (TSMFTIS), and RLG POS. The RLG POS and TSMFTIS were fixed together and then were installed on the inertial stabilized platform. The inertial stabilized platform was fastened in the cabin of airplane. The RLG POS provided attitude of TSMFTIS in remote sensing imaging. The reconstructed spectrum using POS data was better than that from image registration method. The Relative Spectral Quadratic Error (RQE) result demonstrates that the accuracy of the reconstructed spectrum by target-tracking-based method using POS data improves rapidly compared with traditional method. Using the motion solutions measured by 


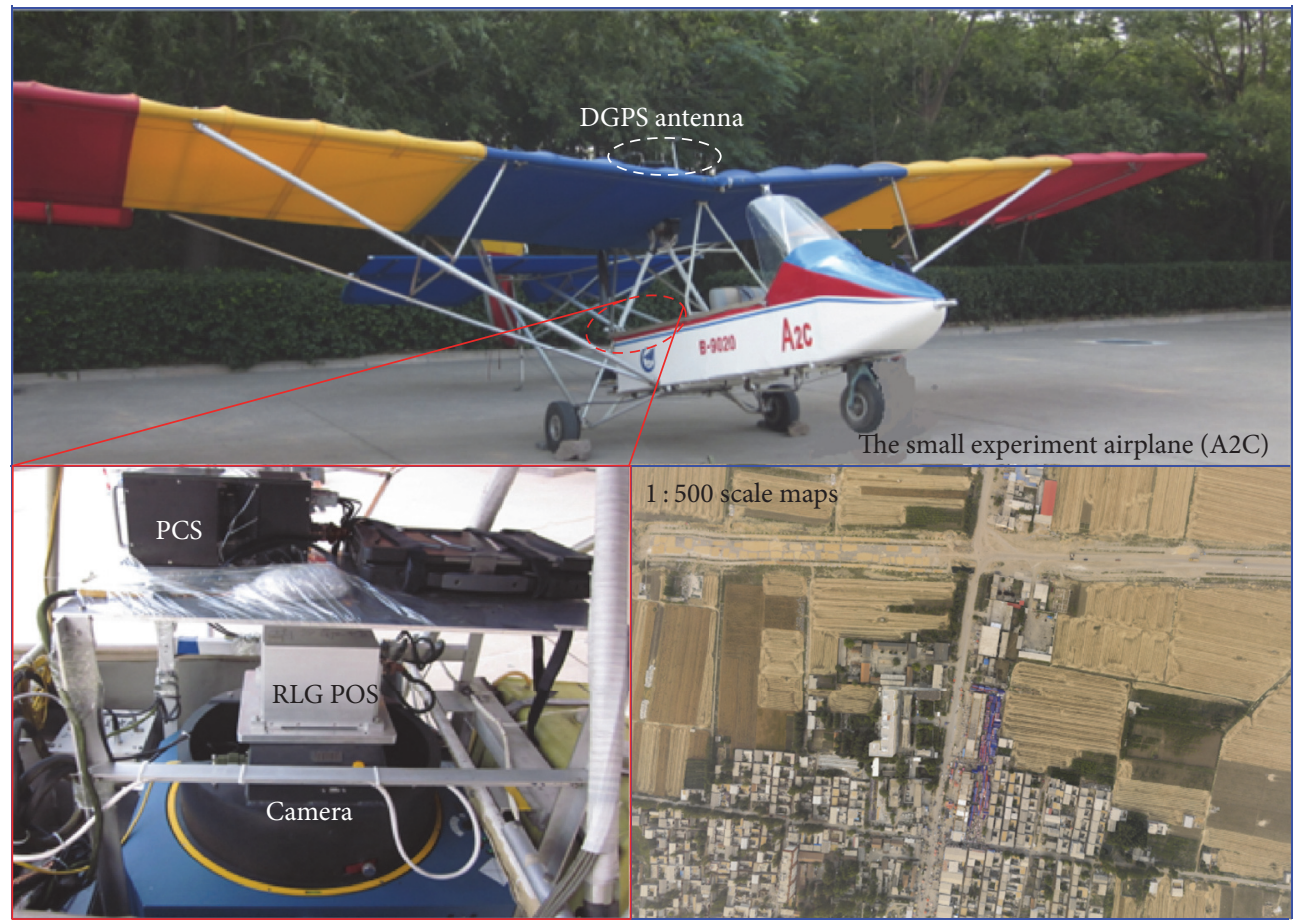

FIgURE 5: The mapping experiment of camera integrated RLG POS.

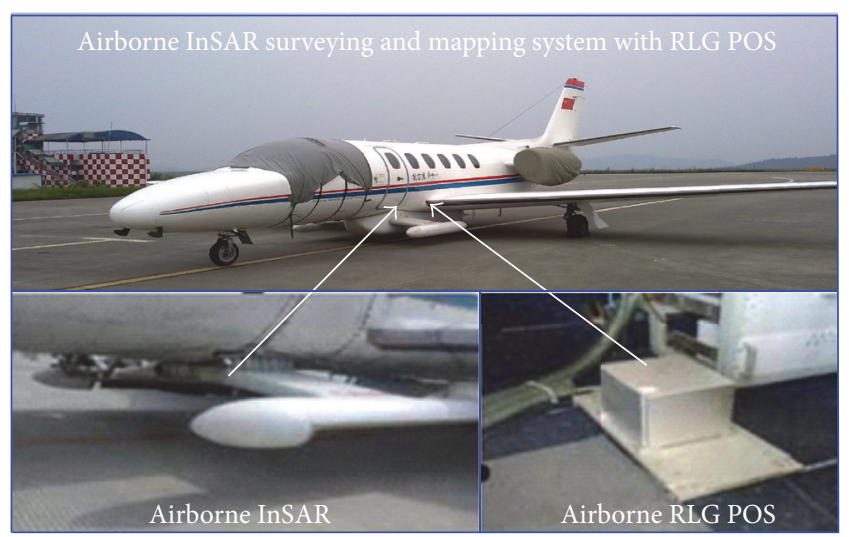

FIGURE 6: POS applied in motion compensation of airborne InSAR.

POS, TSMFTIS successfully achieved aerial remote sensing assignment.

\section{Conclusions}

The airborne POS can precisely measure space-time reference information, has been vital universal equipment, and plays more and more important role of aerial remote sensing system. Some key technologies of airborne POS are summarized. They include the error calibration and compensation, initial alignment, lever arm error modeling, timing synchronization, and integrated estimation method. A few high accuracy POS are developed, have been successfully applied in various

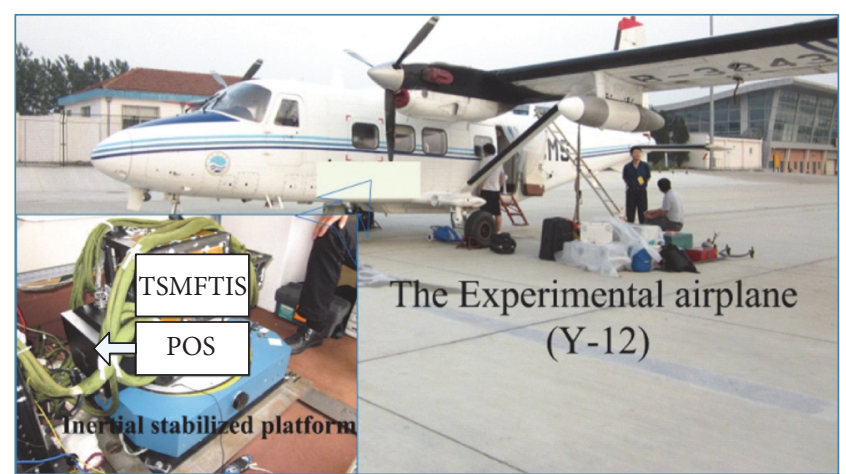

FIgURE 7: The airborne TSMFTIS experiments using POS data.

aerial remote sensing system, and further advance image efficiency and quality of imaging sensors.

\section{Competing Interests}

The authors declare that they have no competing interests.

\section{Acknowledgments}

The work described in the current paper was supported by National Natural Science Foundation of China under Grants 61571030 and 61421063, by the National High Technology Research and Development Program of China (863 program 2015AA124001), and in part by Basic Scientific Research under Grant YWF-16-BJ-Y-30. 


\section{References}

[1] M. Mostafa and J. H. Mostafa, "Direct positioning and orientation systems, How do they work? What is the attainable accuracy?" in Proceedings of the American Society of Photogrammetry and Remote Sensing Annual Meeting, pp. 1-11, St. Louis, Mo, USA, April 2001.

[2] P. Gordon, "Airborne digital data capture systems," in Proceedings of the 7th International Scientific \& Technical Conference, from Imagery to Map: Digital Photogrammetric Technologies, pp. 1-32, Nesebar, Bulgaria, 2007.

[3] Q. Du, B. Xu, and H. Cao, "Registration of airborne LiDAR data and aerial images based on straight lines and POS data," in Proceedings of the Remote Sensing and GIS Data Processing and Other Applications: 6th International Symposium on Multispectral Image Processing and Pattern Recognition (MIPPR '09), pp. 1-6, November 2009.

[4] M. M. R. Mostafa and K.-P. Schwarz, "Digital image georeferencing from a multiple camera system by GPS/INS," ISPRS Journal of Photogrammetry and Remote Sensing, vol. 56, no. 1, pp. 1-12, 2001.

[5] X. Zhong, M. Xiang, H. Yue, and H. Guo, "Algorithm on the estimation of residual motion errors in airborne sar images," IEEE Transactions on Geoscience and Remote Sensing, vol. 52, no. 2, pp. 1311-1323, 2014.

[6] J. Zhang, "Block adjustment of POS-supported airborne SAR images," in Proceedings of the 12th International Radar Symposium, pp. 863-868, Leipzig, Germany, September 2011.

[7] T. A. Kennedy, "The design of SAR motion compensation systems incorporation strapdown inertial measurement unit," in Proceedings of the IEEE National Radar Conference, pp. 7478, 1988.

[8] E. Lithopoulos, B. Reid, and B. Scherzinger, "The position and orientation system (POS) for survey applications," in Proceedings of the International Archives of Photogrammetry and Remote Sensing, pp. 467-471, Vienna, Austria, 1996.

[9] D. Li, Q. Tong, R. Li, J. Gong, and L. Zhang, "Current issues in high-resolution earth observation technology," Science China Earth Sciences, vol. 55, no. 7, pp. 1043-1051, 2012.

[10] M. Mostafa, J. Hutton, and B. Reid, "GPS/IMU products the Applanix approach," in Proceedings of the Photogrammtric Week 01, pp. 63-83, Wichmann, Stuttgart, Germany, 2001.

[11] M. Mostafa and J. Hutton, "Airborne remote sensing without ground control," in Proceedings of the International Geoscience and Remote Sensing Symposium (IGARSS '01), pp. 2961-2963, Sydney, Australia, July 2001.

[12] A. W. L. Ip, N. El-Sheimy, and M. Mostafa, "System performance analysis of INS/DGPS integrated system for mobile mapping system(MMS)," in Proceedings of the 4th International Symposium on Mobile Mapping Technology, pp. 1-10, Kunming, China, April 2004.

[13] T. Dautermann, "Civil air navigation using GNSS enhanced by wide area satellite based augmentation systems," Progress in Aerospace Sciences, vol. 67, pp. 51-62, 2014.

[14] A. Frank and J. Hutton, "GPS and inertial navigationdelivering," GEOconnexion International Magazine, no. 9, pp. 52-53, 2005.

[15] S. E. Dmitriyev, O. A. Stepanov, and S. V. Shepel, "Nonlinear filtering methods application in INS alignment," IEEE Transactions on Aerospace and Electronic Systems, vol. 33, no. 1, pp. 260-272, 1997.
[16] R. Zhao and Q. Gu, "Nonlinear filtering algorithm with its application in INS alignment," in Proceedings of the 10th IEEE Workshop on Statistical Signal and Array Processing, pp. 510-513, Pocono Manor, Pa, USA, August 2000.

[17] A. H. Mohamed and K. P. Schwarz, "Adaptive Kalman filtering for INS/GPS," Journal of Geodesy, vol. 73, no. 4, pp. 193-203, 1999.

[18] S. Särkkä and J. Hartikainen, "On Gaussian optimal smoothing of non-linear state space models," IEEE Transactions on Automatic Control, vol. 55, no. 8, pp. 1938-1941, 2010.

[19] S. Särkkä, "Unscented rauch-tung-striebel smoother," IEEE Transactions on Automatic Control, vol. 53, no. 3, pp. 845-849, 2008.

[20] T. Beravs, J. Podobnik, and M. Munih, “Three-axial accelerometer calibration using kalman filter covariance matrix for online estimation of optimal sensor orientation," IEEE Transactions on Instrumentation and Measurement, vol. 61, no. 9, pp. 2501-2511, 2012.

[21] X. Gong and R. Zhang, "Analysis the effect of IMU calibration errors on SINS/GPS integration accuracy for airborne earth observation," in Proceedings of the 3rd International Symposium on Information Engineering and Electronic Commerce (IEEC '11), pp. 38-42, Huangshi, China, July 2011.

[22] J. Li, F. Jiao, J. Fang, and Y. Ma, "Integrated calibration method for dithered RLG POS using a hybrid analytic/Kalman filter approach," IEEE Transactions on Instrumentation and Measurement, vol. 62, no. 12, pp. 3333-3342, 2013.

[23] C. Shen and X. Chen, "Analysis and modeling for fiber-optic gyroscope scale factor based on environment temperature," Applied Optics, vol. 51, no. 14, pp. 2541-2547, 2012.

[24] H.-S. Ahn, C.-H. Won, D. Olsen et al., "Initial attitude estimation of tactical grade inertial measurement unit for airborne environmental camera," in Proceedings of the American Control Conference, pp. 4403-4408, June 2003.

[25] M. Wu, Y. Wu, X. Hu, and D. Hu, "Optimization-based alignment for inertial navigation systems: theory and algorithm," Aerospace Science and Technology, vol. 15, no. 1, pp. 1-17, 2011.

[26] S. Lü, L. Xie, and J. Chen, "New techniques for initial alignment of strapdown inertial navigation system," Journal of the Franklin Institute, vol. 346, no. 10, pp. 1021-1037, 2009.

[27] J. Li, J. Fang, and M. Du, "Error analysis and gyro-bias calibration of analytic coarse alignment for airborne POS," IEEE Transactions on Instrumentation and Measurement, vol. 61, no. 11, pp. 3058-3064, 2012.

[28] Y. Ma, J. Fang, and J. Li, "Accurate estimation of lever arm in SINS/GPS integration by smoothing methods," Measurement, vol. 48, no. 1, pp. 119-127, 2014.

[29] Q. Cao, M. Zhong, and Y. Zhao, "Dynamic lever arm compensation of SINS/GPS integrated system for aerial mapping," Measurement, vol. 60, pp. 39-49, 2015.

[30] Y. Ma, X. Mai, and K. Wang, "Changing dimensional feedback correction method of INS/GPS integrated navigation system based on lever arm estimation," in Proceedings of the 3rd International Conference on Advanced Engineering Materials and Architecture Science (ICAEMAS '14), pp. 651-653, Hohhot, China, July 2014.

[31] Y. J. Cui, S. G. Shuzhi, and T. Goh, "Synchronization solutions for a loosely INS and GPS navigation system," in Proceedings of the Asian Control Conference, pp. 1816-1821, Singapore, September 2002. 
[32] K. Kim and C. G. Park, "Drift error analysis caused by RLG dither axis bending," Sensors and Actuators A, vol. 133, no. 2, pp. 425-430, 2007.

[33] J. Li, A. Chen, J. Fang, and J. Cheng, "Time delay modeling and compensation of dithered RLG POS with antivibrators and filter," Measurement: Journal of the International Measurement Confederation, vol. 46, no. 6, pp. 1928-1937, 2013.

[34] J. Fang and X. Gong, "Predictive iterated kalman filter for INS/GPS integration and its application to SAR motion compensation," IEEE Transactions on Instrumentation and Measurement, vol. 59, no. 4, pp. 909-915, 2010.

[35] Y. Xu, W. Sun, and P. Li, "A miniature integrated navigation system for rotary-wing unmanned aerial vehicles," International Journal of Aerospace Engineering, vol. 2014, Article ID 748940, 13 pages, 2014.

[36] X. Gong and T. Qin, "Airborne earth observation positioning and orientation by SINS/GPS integration using CD R-T-S smoothing," Journal of Navigation, vol. 67, no. 2, pp. 211-225, 2014. 


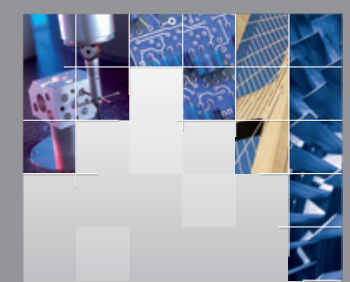

\section{Enfincering}
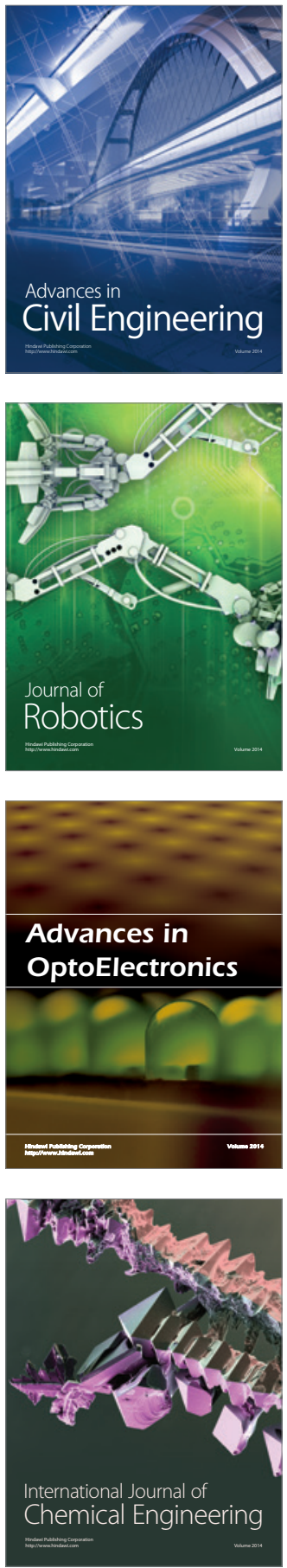

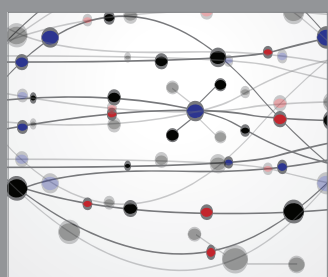

The Scientific World Journal

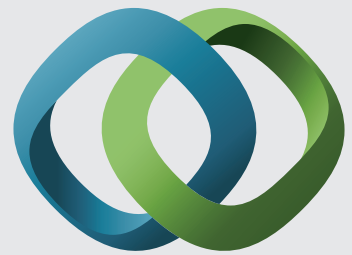

\section{Hindawi}

Submit your manuscripts at

https://www.hindawi.com
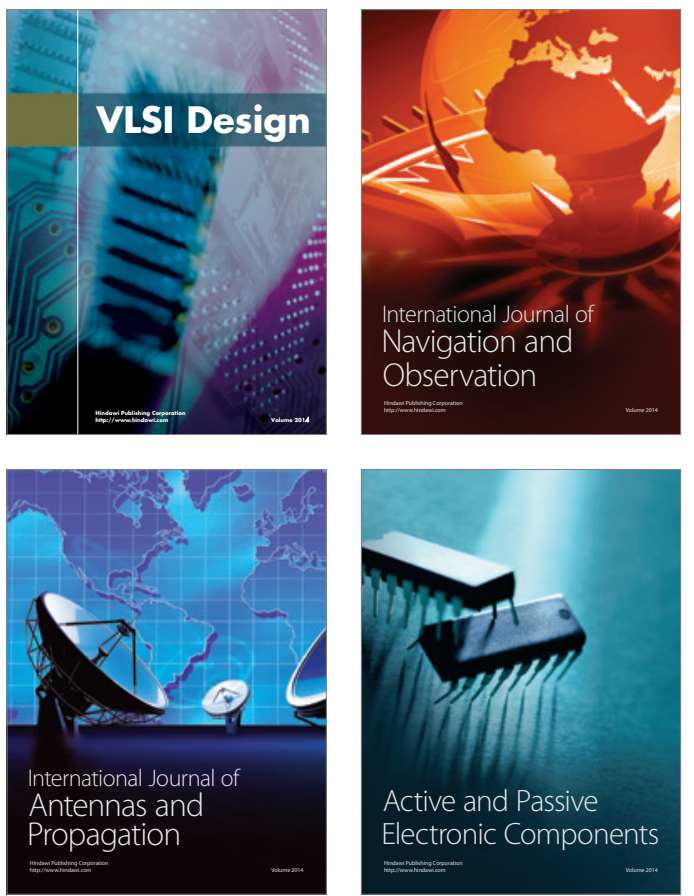
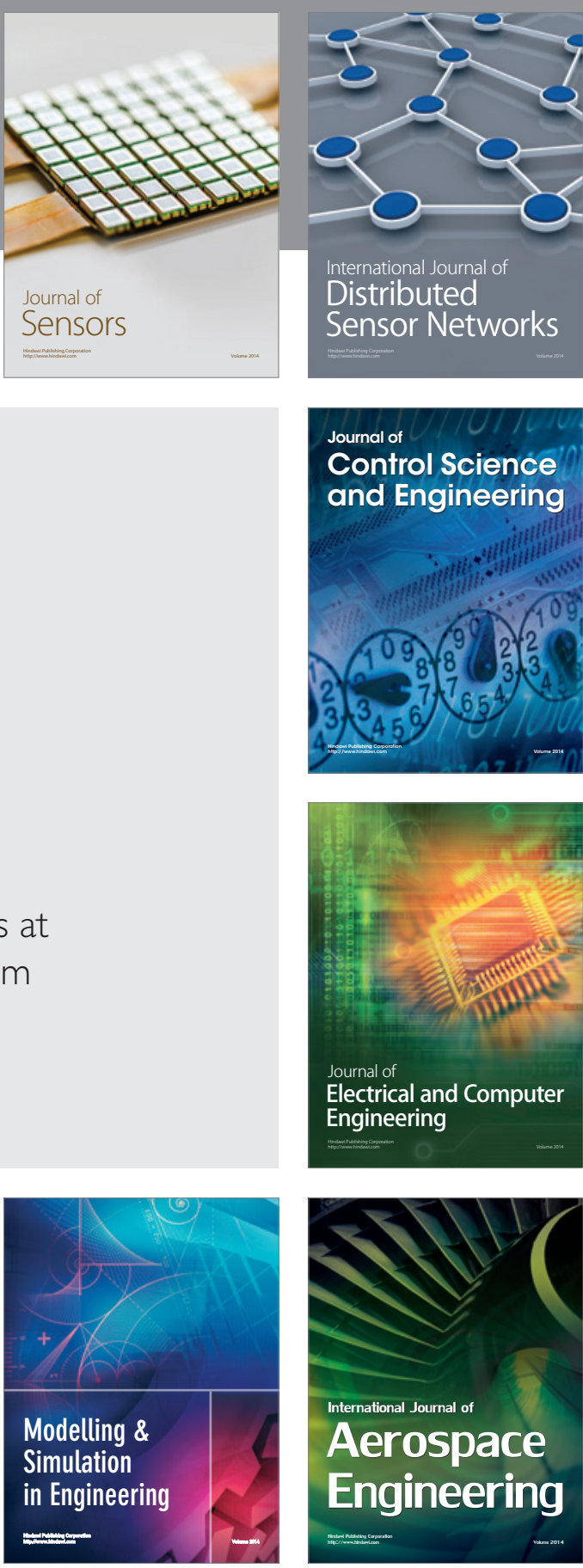

International Journal of

Distributed

Sensor Networks

$-$

Joumal of

Control Science

and Engineering
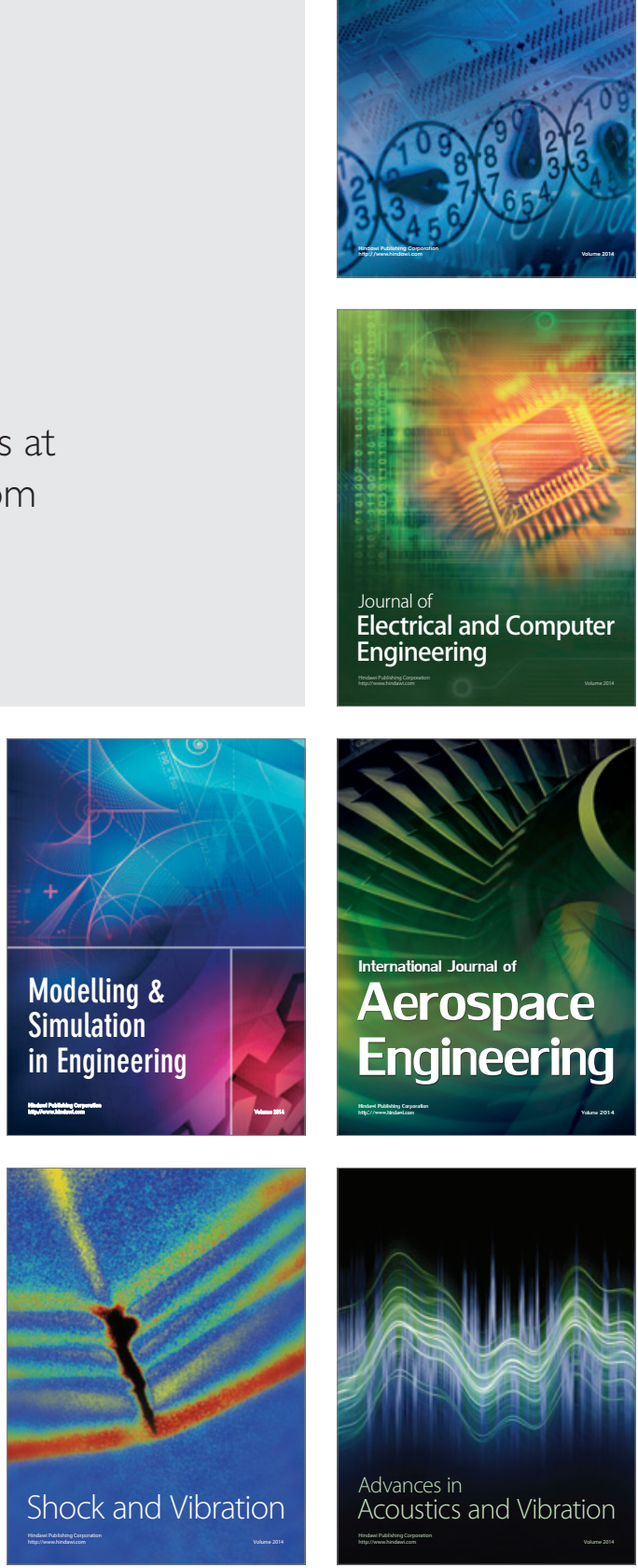